\title{
Prolonged hepatitis and jaundice: a rare complication of paediatric Epstein-Barr virus infection
}

\author{
Zhen Han $\underline{T a n}^{1}$, MBBS, MRCPCH, Kong Boo $\underline{\text { Phua }}^{1}$, FRCPE, FRCPCH, Christina $\underline{\text { Ong }}^{1}$, FRCPCH, CCT, Ajmal Kader ${ }^{1}$, FRCPCH, CCT
}

\begin{abstract}
We herein report the case of a 14-year-old girl with Epstein-Barr virus (EBV) infectious mononucleosis who developed prolonged hepatitis and jaundice. At presentation, she had tender hepatomegaly with a markedly deranged liver function test. Abdominal ultrasonography showed hepatomegaly and a thickened gallbladder wall. During the subsequent 11 weeks, her transaminases showed two further peaks, which corresponded with clinical deterioration. Her highest alanine transaminase level was $1,795 \mu / L$ and total bilirubin level was $154 \mu \mathrm{mol} / \mathrm{L}$. She recovered fully with conservative management. EBV-related liver involvement is typically mild and self-limiting. We believe that tender hepatomegaly and gallbladder thickening may be important predictors of significant liver involvement. Although multiple transaminase peaks may occur, we do not consider this an indication for antiviral or immunosuppressive therapy. In the absence of strong evidence supporting the use of any specific therapy, we recommend a conservative approach for an immunocompetent patient.
\end{abstract}

Keywords: Epstein-Barr virus, hepatitis, jaundice, mononucleosis, paediatric

\section{INTRODUCTION}

Epstein-Barr virus (EBV) is a common paediatric infection that has seropositivity rates of $90 \%-95 \%$ by adulthood. ${ }^{(1-3)}$ The majority of EBV infections in childhood are asymptomatic. However, young adolescents tend to experience symptomatic disease in the form of infectious mononucleosis (IM). (1-3) $^{-3}$

Hepatic involvement in EBV infections is usually mild and self-limiting. ${ }^{(1,3,4)}$ Elevated hepatic transaminase levels are typically less than five times the upper limit of normal levels ${ }^{(1,4)}$ and rarely exceed 1,000 U/L. ${ }^{(3)}$ Jaundice, which has been reported in up to $5 \%$ of cases, is due to haemolysis or cholestasis ${ }^{(1)}$ and is less frequently seen in children than adults. ${ }^{(5)}$ To the best of our knowledge, there are no reliable parameters that predict significant liver involvement. We herein report a case of paediatric EBV infection associated with prolonged hepatitis and jaundice.

\section{CASE REPORT}

A previously healthy 14-year-old Chinese girl presented with a four-day history of high-grade fever associated with sore throat, epigastric pain and decreased appetite. Clinically, she was febrile and lethargic. Physical examination showed cervical lymphadenopathy, pharyngeal injection, bilaterally enlarged tonsils and tender hepatomegaly. She was not jaundiced and the spleen was not palpable.

A full blood count showed lymphocyte-predominant leucocytosis with 7\% atypical lymphocytes. The liver function test (LFT) showed mild elevation of bilirubin of 41 (normal range [NR] 13-20) $\mu \mathrm{mol} / \mathrm{L}$, with direct bilirubin of 34 (NR 2-7) $\mu \mathrm{mol} / \mathrm{L}$, and elevated alanine transaminase (ALT) of 658 (NR 8-22) U/L, as well as aspartate transaminase of 313 (NR 13-26) U/L. Alkaline phosphatase and gamma glutamyl transferase levels were also elevated at 282 (NR 62-280) U/L and 230 (NR 7-21) U/L, respectively. Abdominal ultrasonography showed hepatomegaly measuring $17.8 \mathrm{~cm}$ vertically and marked gallbladder wall thickening measuring $15 \mathrm{~mm}$ (normal $<3 \mathrm{~mm}$ ) with a striated appearance (Fig. 1). Screening for hepatotropic viruses was negative for Hepatitis A, B and C, while serologies for cytomegalovirus (CMV) and EBV were positive for immunoglobulin M (IgM) and immunoglobulin G (IgG) antibodies. Results of a blood test for CMV polymerase chain reaction (PCR) were negative. C-reactive protein test results and blood cultures were negative.

The diagnosis of IM with EBV hepatitis was made and the patient was managed conservatively. A few doses ( $<60 \mathrm{mg} / \mathrm{kg} /$ day) of acetaminophen were administered on the initial three days, with no other drug ingestion. There was clinical and biochemical improvement by the end of the first week. However, symptoms returned on Day 16 with low-grade fever, extreme lethargy, poor appetite, weight loss and jaundice. The patient's LFT showed markedly raised transaminases and conjugated hyperbilirubinaemia (Fig. 2a \& b). Her LFTs were closely monitored and she was commenced on ursodeoxycholic acid as well as multivitamins. In view of the significant transaminitis, diagnostic workup was performed for other causes of hepatitis. Extended screening investigations were negative for autoimmune hepatitis, Wilson's disease and haemophagocytic lymphohistiocytosis $(\mathrm{HLH})$. After a peak in transaminases (ALT 1,795 U/L) on Day 18, an improving trend followed, only to worsen again with a third peak (ALT $815 \mathrm{U} / \mathrm{L}$ ) on Day 40. By Day 80, transaminases returned to normal. The trend of rising bilirubin levels lagged behind that of the transaminases, peaking at $154 \mu \mathrm{mol} / \mathrm{L}$ on Day 29 (Fig. 2a). A liver biopsy was held off as liver

${ }^{1}$ Department of Paediatric Medicine, KK Women's and Children's Hospital, Singapore

Correspondence: Dr Kader Ajmal, Consultant, Department of Paediatric Medicine, KK Women's and Children's Hospital, Level 3, Children's Tower, 100 Bukit Timah Road, Singapore 229899. ajmalkader@hotmail.com 


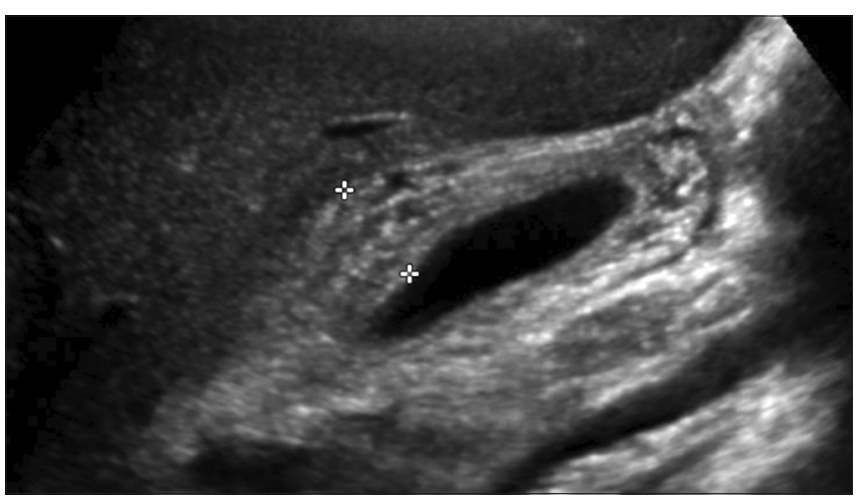

Fig. 1 Abdominal US image shows thickened gallbladder wall measuring $15 \mathrm{~mm}$ (indicated by the plus signs) with a striated appearance.

synthetic function remained good with no significant alteration in serum albumin or coagulation profile. Repeat abdominal ultrasonography on Day 25 showed complete resolution of the hepatomegaly and gallbladder wall thickening. Blood test results for EBV PCR on Day 40 were negative.

Clinically, the patient was continuously febrile for three weeks and had intermittent low-grade fever till the sixth week. Clinical jaundice was noted after the second week and lasted till the sixth week. The patient felt unwell with anorexia and lethargy during a period that corresponded with the transaminase peaks. Her documented weight loss was $5 \mathrm{~kg}$ over the initial six weeks. At a three-month review, she had completely recovered with normal LFT.

\section{DISCUSSION}

For EBV, like other members of the Herpesviridae family, infection typically spreads via direct contact with oral secretions. Its incubation period is 5-7 weeks and the illness lasts for a median duration of 16 days when it is symptomatic. EBV infection most commonly occurs in early childhood with a second peak in late adolescence. The principal cause of IM is primary EBV infection and most people recover without sequelae. A large number of clinical manifestations have been associated with EBV infection, including hepatitis, haemolytic anaemia, thrombocytopenia, splenic rupture and myocarditis.(1) Rare life-threatening complications in apparently immunocompetent patients include $\mathrm{HLH}$ and chronic active EBV (CAEBV) infection. ${ }^{(2)}$

In our case, the diagnosis of EBV infection was reached based on a clinical picture that was consistent with IM, and detection of EBV viral capsid antigen (VCA) IgM and IgG in the serum. We did not perform the serum test for EBV nuclear antigen-1 (EBNA-1) IgG, which is indicative of past infection. ${ }^{(6)}$ VCA IgM is not detected in chronic infection; thus, its presence is virtually diagnostic of primary EBV infection. ${ }^{(2)}$ Using all the three serological parameters, VCA IgM, VCA IgG and EBNA-1 IgG, improves the ability to distinguish between acute and past infections in immunocompetent patients. ${ }^{(6)}$ The serological profile of a positive VCA IgM and IgG together with a negative EBNA-1 IgG indicates acute infection. ${ }^{\left({ }^{6}\right)}$ As previously proposed, serology can be considered the gold standard for diagnosis of primary EBV infection in immunocompetent patients. ${ }^{(7)}$ Our patient's test results for CMV IgM and IgG were also positive, suggesting the possibility of co-infection of EBV and CMV. In such cases, longer clinical courses have been reported. ${ }^{(8)}$ However, the CMV IgM test was likely to be a false positive as results of a blood test for CMV PCR conducted a few days later were negative. Due to the patient's clinical recovery, a convalescent CMV IgG test was not performed. False-positive results for CMV IgM in EBV infection are thought to be due to cross-reactions between major antigenic epitopes. ${ }^{(9)}$

In EBV infection, hepatic involvement is seen in up to $80 \%-90 \%$ of cases. ${ }^{(1,3,4)}$ Hepatomegaly and hepatic tenderness have been reported in $10 \%-15 \%$ of cases. ${ }^{(1)}$ Frequently, the only evidence of hepatic involvement is transaminitis, with transaminases that are often less than five times of normal levels. ${ }^{(1,4)}$ Transaminitis usually occurs during the second to fourth week of illness and normalises within six weeks. ${ }^{(1)}$ However, as seen in our case, transaminitis occurring in the first week has also been reported. (3) A literature review of paediatric cases of IM from Southeast Asia revealed that up to $60 \%$ of patients had transaminitis. Incidence increased with age and peak transaminase levels were usually below $500 \mathrm{U} / \mathrm{L} .{ }^{(10)}$ Our patient, who had no pre-existing liver disease or predisposition, had transaminitis from the first week of illness. Following an initial improvement, serum transaminases peaked again between the second and third weeks, with levels exceeding 1,750 U/L. After another brief period of improvement, a third peak was noted between the fifth and sixth weeks. A similar pattern of multiple peaks in transaminases has been reported in 41 adult patients by Kofteridis et al. However, the peaks occurred within the initial two weeks and normalised by the third week in that study. ${ }^{(4)}$ The underlying reason for multiple transaminase peaks is unknown. In approximately $5 \%$ of all cases, mild jaundice was noted, which may result from haemolysis or cholestasis. ${ }^{(1)}$ Jaundice is seen less frequently in children than adults. ${ }^{(5)}$ After the second week, our patient developed clinical jaundice and conjugated hyperbilirubinaemia due to cholestasis. The bilirubin reached a peak of $154 \mu \mathrm{mol} / \mathrm{L}$ in the fourth week of illness, a level that is rare in children in the absence of haemolysis. ${ }^{(11)}$

Sonographic findings in EBV hepatitis include nonspecific features such as hepatomegaly, splenomegaly, porta hepatis adenopathy, periportal oedema ${ }^{(12)}$ and gallbladder wall thickening. ${ }^{(13)}$ The association between gallbladder wall thickening and EBV hepatitis has been posited as a sign of the severity of the hepatitis. ${ }^{(13)}$ Notably, our patient had significant gallbladder wall thickening of $15 \mathrm{~mm}$. The underlying mechanism may be an immunological reaction similar to that of hepatitis or gallbladder wall oedema due to lymphatic obstruction from the enlarged porta hepatis lymph nodes. ${ }^{(13)}$

EBV can infrequently cause CAEBV infection in hosts that appear immunocompetent. CAEBV is characterised by chronic or recurrent IM-like symptoms lasting more than six months and an abnormal EBV-related antibody titre or high viral loads. ${ }^{(14)}$ It is associated with a high mortality and morbidity due to life-threatening complications such as haemophagocytic syndrome, coronary artery aneurysms, lymphoma and interstitial 

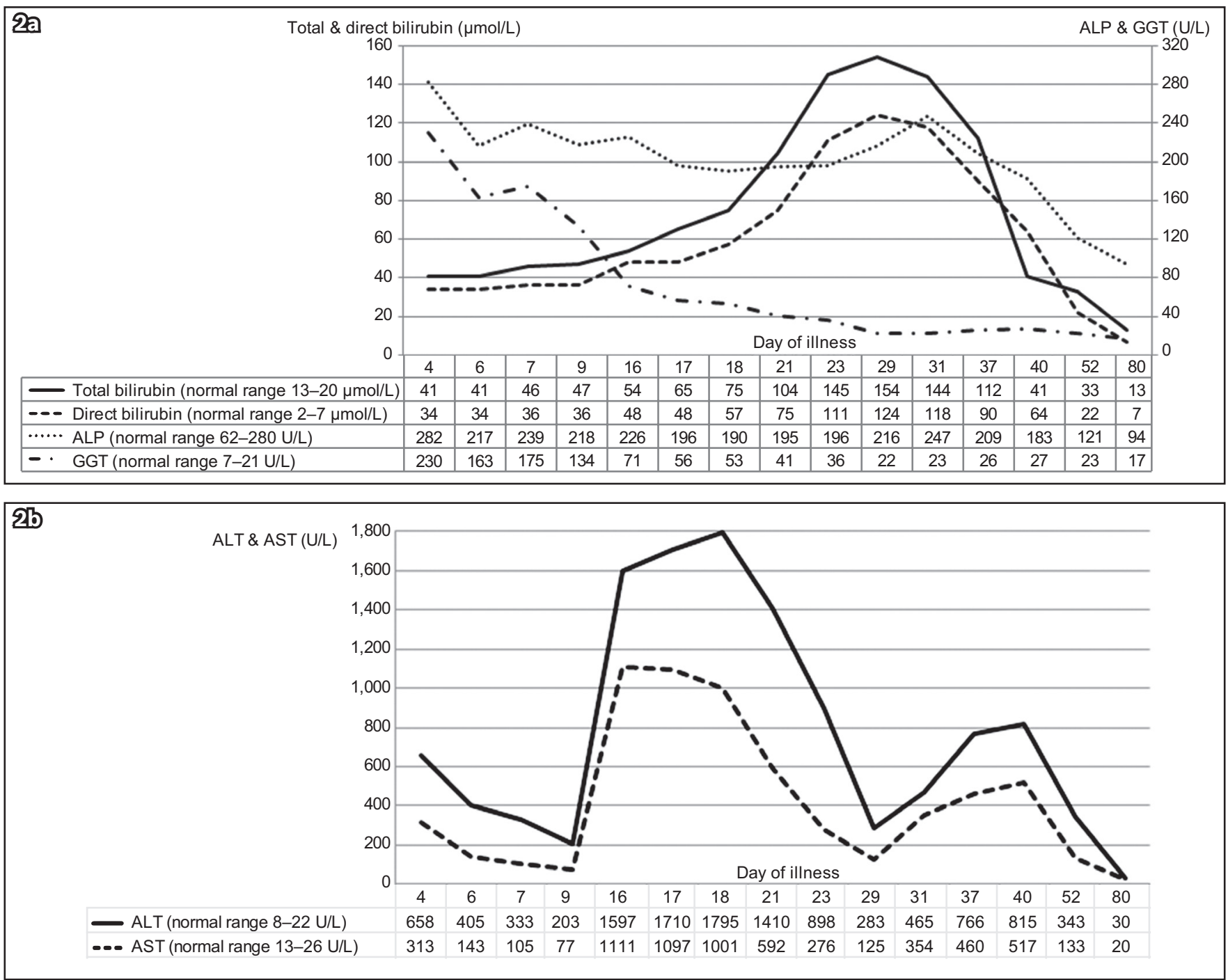

Fig. 2 Graphs of serial LFT results over 11 weeks show changes in (a) total and direct bilirubin, alkaline phosphatase (ALP), and gamma glutamyl transferase (GGT); and (b) serum hepatic transaminases, alanine transaminase (ALT) and aspartate transaminase (AST) as the patient's illness progressed.

pneumonia. ${ }^{(14)}$ Various treatment strategies like antivirals, chemotherapy, immunomodulating agents and cell therapy with EBV-specific cytotoxic T lymphocyte have been attempted. However, they are often unsuccessful unless a haematopoietic stem cell transplant is also performed. ${ }^{(14)}$

In patients with acute primary EBV infection, fulminant liver failure is the leading cause of death, although severe liver injury is rare. ${ }^{(15)}$ Most cases of fulminant liver failure are seen in immunodeficient patients. In immunocompetent subjects, treatment for EBV hepatitis is supportive. Corticosteroids and antivirals including acyclovir and ganciclovir have been used anecdotally, but there is a lack of strong evidence to support their routine use. ${ }^{(4)}$

In conclusion, hepatic involvement in EBV infection typically results in a mild, self-limiting hepatitis. However, EBV infection can, in rare cases, cause significant jaundice as well as prolonged hepatitis with multiple serum transaminase peaks. Close monitoring during the clinical course of the illness is recommended, as a minority of patients may develop acute liver failure or progress to CAEBV. In the absence of immunodeficiency, treatment of EBV-associated hepatitis remains supportive.

\section{REFERENCES}

1. Jenson HB. Acute complications of Epstein-Barr virus infectious mononucleosis. Curr Opin Pediatr 2000; 12:263-8.

2. Luzuriaga K, Sullivan JL. Infectious mononucleosis. N Engl J Med 2010; 362:1993-2000

3. Vine LJ, Shepherd K, Hunter JG, et al. Characteristics of Epstein-Barr virus hepatitis among patients with jaundice or acute hepatitis. Aliment Pharmacol Ther 2012; 36:16-21.

4. Kofteridis DP, Koulentaki M, Valachis A, et al. Epstein Barr virus hepatitis. Eur J Intern Med 2011; 22:73-6.

5. Sumaya CV, Ench Y. Epstein-Barr virus infectious mononucleosis in children. I. Clinical and general laboratory findings. Pediatrics 1985; 75:1003-10.

6. De Paschale M, Clerici P. Serological diagnosis of Epstein-Barr virus infection: Problems and solutions. World J Virol 2012; 1:31-43.

7. Hess RD. Routine Epstein-Barr virus diagnostics from the laboratory perspective: still challenging after 35 years. J Clin Microbiol 2004; 42:3381-7.

8. Wang X, Yang K, Wei C, Huang Y, Zhao D. Coinfection with EBV/ $\mathrm{CMV}$ and other respiratory agents in children with suspected infectious mononucleosis. Virol J 2010; 7:247.

9. Park JM, Shin JI, Lee JS, et al. False positive immunoglobulin m antibody to cytomegalovirus in child with infectious mononucleosis caused by Epstein-Barr virus infection. Yonsei Med J 2009; 50:713-6.

10. Chan CW, Chiang AK, Chan KH, Lau AS. Epstein-Barr virus-associated infectious mononucleosis in Chinese children. Pediatr Infect Dis J 2003; 22:974-8.

11. Chambers CV, Irwin CE Jr. Intense jaundice in an adolescent. An unusual 
presentation of infectious mononucleosis. J Adolesc Health Care 1986; 7:195-7.

12. Crum NF. Epstein Barr virus hepatitis: case series and review. South Med J 2006; 99:544-7.

13. O'Donovan N, Fitzgerald E. Gallbladder wall thickening in infectious mononucleosis: an ominous sign. Postgrad Med J 1996; 72:299-300.
14. Kimura H. Pathogenesis of chronic active Epstein-Barr virus infection: is this an infectious disease, lymphoproliferative disorder, or immunodeficiency? Rev Med Virol 2006; 16:251-61.

15. Feranchak AP, Tyson RW, Narkewicz MR, Karrer FM, Sokol RJ. Fulminant Epstein-Barr viral hepatitis: orthotopic liver transplantation and review of the literature. Liver Transpl Surg 1998; 4:469-76. 